\title{
3D Quantification Visualization of Vascular Structures in Magnetic Resonance Angiographic Images
}

\author{
J.A. Schaap MSc., P.J.H. de Koning MSc., J.P. Janssen MSc., J.J.M. \\ Westenberg PhD., R.J. van der Geest MSc., and J.H.C. Reiber PhD. \\ Division for Image Processing, Dept. of Radiology, Leiden University Medical Center, \\ Leiden, the Netherlands j.a.schaap@lumc.nl
}

\begin{abstract}
This paper describes a new method to segment vascular structures in 3D MRA data, based on the Wavefront Propagation algorithm. The center lumen line and the vessel boundary are detected automatically. Our 3D visualization and interaction platform will be prestended, which is used to aid the phycisian in the analysis of the MRA data. The results are compared to conventional X-ray DSA which is considered the current gold-standard. Provided that the diameter of the vessel is larger than 3 voxels, our method has similar result as X-ray DSA.
\end{abstract}

\section{Introduction}

Determination of vessel morphology along a segment of a vessel is important in grading vascular stenosis. Until recently most assessments of vascular stenosis were carried out using X-Ray Digital Subtraction Angiography (X-DSA). This technology has proven itself in the assessment of stenoses. However several problems exist when using this technology. Because X-DSA is a projection technique, over-projection of different vessels can occur even if the viewing angle is set optimally. Additionally, a contrast agent has to be injected, which requires an invasive procedure.

Magnetic Resonance Angiography (MRA) is a technique which acquires three dimensional (3D) images of vascular structures and the surrounding anatomy. Because of the 3D nature of the images, there is no problem of over-projection of different vessels. MRA images can be obtained without using a contrast agent, however by using a contrast agent the contrast-to-noise ratio increases. This contrast agent is normally applied intra-venously.

At the present time, evaluation of MRA images is commonly performed on two-dimensional (2D) Maximum Intensity Projections (MIPs), although it is known that this leads to under-estimation of the vessel diameter and a decreased contrast-to-noise ratio [2]. To improve upon the conventional analysis of MRA, it would be desirable to obtain quantitative morphological information directly from the $3 \mathrm{D}$ images and not from the MIPs. To accomplish this, accurate 3D 
segmentation tools are required. Vessel segmentation of 3D images has been investigated by many researchers. However the majority of this research focussed on enhancing the $3 \mathrm{D}$ visualization of the vascular structures in the image and not on accurate quantification of these structures.

In this paper we describe a novel approach for quantitative vessel analysis of MRA images. Our approach uses knowledge about the image acquisition to accurately determine the vessel boundaries. The techniques we use operates on the full 3D images, and not the projections.

This paper is organized as follows. In Section 2 we present the algorithms which we use to segment a vessel, and the visualization platform is described. In Section 3 we describe the methods and materials we used for the validation of our approach. We conclude this paper with the discussion, future work and conclusions.

\section{Methods}

The focus of this section will be on the general outline of the used algorithms, and on the interaction between the user, the data and the algorithms. More information on the algorithms can be found in the references.

In short, the analysis trajectory is as follows: After loading and visually inspecting the data, the user places two points at the beginning and at the end of the vessel segment of interest. Using wavefront propagation and backtracking, a minimal cost path is found between these points through the vessel. This minimal cost path is then adjusted to form the center-lumen path. A tubular model constructed of a NURBS-surface is placed around the center-lumen path. The surface is then fitted to the data by balancing a set of internal and external forces that act on the surface. The result is presented visually, in graphs and can be exported to tables. The platform also provides means to generate snapshots and movies of any stage in the analysis. It should be noted that the only required user input is the placement of the two points in the vessel. The rest of the method is completely automated, thus minimizing inter- and intra user variability. We will now look into the different parts in more depth.

\subsection{Wavefront Propagation}

The 3D pathline detector is based on the Fast Marching Level Set Method (FMLSM) as described by Sethian et al.[7,8,5,4]. The FMLSM calculates the propagation of a wave through a medium. Starting from one (or more) given location the propagation of the wave is calculated until a certain stop criterium is met. The stop criterium in most cases is the reaching of a given end point, however the propagation can continue until every point has been reached.

If we think of the image as an inhomogeneous medium, then the wave propagates through some parts of the image faster than through other parts. The idea is to make the wave propagate fast through the vessel, and slow through 


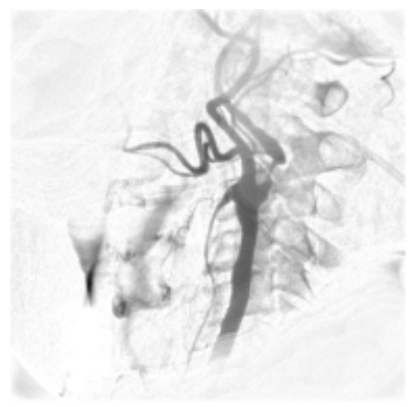

(a) A filtered X-ray image of the coronary arteries.

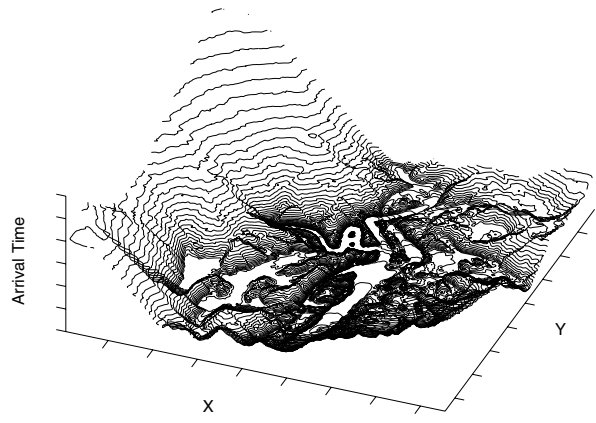

(b) $T$ surface after the wave has propagated. The starting point is located in the bottom right part of the image

Fig. 1. Example of the use of the WaveProp module.

the rest of the image. This can be done by making the speed dependant on local image information like image intensity or image gradient.

Figure 1(a) and 1(b) shows an example: 1(a) shows the original image and 1 (b) shows the associated T-surface. This surface represents the time at which the wave passed each point $(x, y)$. An inverse linear speed function $\operatorname{speed}(x, y)=$ $\operatorname{Max}-I(x, y)$ is used, where $I(x, y)$ is the image intensity at location $(x, y)$, and where Max is the maximum intensity of the whole image. Therefore the wave will propagate faster through darker regions (i.e. the vessels) which translates itself in the valleys in the T-surface.

\subsection{Back tracking}

In order to find the path between two points $p_{0}$ and $p_{1}$, we use the arrival times that have been calculated by the FMLSM. Every point that has been reached during the propagation has an arrival time -the time at which the wavefront arrived at this point- associated with it. In figure $1(\mathrm{~b})$ the arrival times of a $2 \mathrm{D}$ $\mathrm{X}$-ray image are plotted as so-called T-surface. Using a steepest descent approach we move from $\left(p_{1}, T\left(p_{1}\right)\right.$ to $\left(p_{0}, 0\right)$. The surface $\mathrm{T}$ has a convex like behavior in the sense that starting from any point $(p, T(p))$ on the surface and following the gradient descent direction, we will always converge to $p_{0}$. Given the point $p_{1}$, the path connecting $p_{0}$ (the point in $T$ with the smallest value) and $p_{1}$, called the minimal path, is the curve $\widetilde{\mathcal{C}}(\sigma)$ starting at $\widetilde{\mathcal{C}}(0)=p_{1}$ and following the opposite gradient direction on $T$ :

$$
\frac{\partial \widetilde{\mathcal{C}}}{\partial \sigma}=-\nabla T
$$


The back-tracking procedure is a simple steepest gradient descent approach. It is possible to make a simple implementation on a rectangular grid: given a point $q=(i, j)$, the next point in the chain connecting $q$ to $p$ is selected to be the grid neighbor $(k, l)$ for which $T(k, l)$ is the minimal, and so forth.

\subsection{Speed function}

Since the speed function is essential to the algorithm, any manual adjustment in this speed function, may result in inter- and intra- user variability. Therefore, the speed function required by the FMLSM is based on the global image histogram. A sigmoid function is chosen for the speed function. The function is controlled by two parameters. $\mathrm{L}_{50}$ varies the position where the speed is $50 \%$ of the maximum speed $\left(\mathrm{F}_{M A X}\right)$ and slope modifies the slope of the speed function. $\mathrm{F}_{M A X}$ is set to 100 .

\subsection{Path correction}

The minimal path obtained by the back tracking algorithm tends to cut the corners. We are interested however in the path through the center of the lumen. To correct the minimal path to form the center lumen line we use a modified Boundary Surface Shrinking (BSS) method [6]. This methods moves points along the gradient of a distance map [3]. The distance map contains the minimal distance to the object's boundary for every voxel, measured in some suitable metric. The gradient of the distance map at a point $x$ will simply be denoted by $\nabla D(x)$ throughout this text.

The key idea of the original BSS algorithm is to iteratively translate the vertices of the initial surface along the gradient vectors at their current positions. Our modified version moves the points of our minimal path, instead of the vertices of the boundary surface.

The position of point $i$ in iteration $n+1$ is given by:

$$
x_{i}^{(n+1)}:=x_{i}^{(n)}+h \nabla D\left(x_{i}^{(n)},\right)
$$

where $h$ determines the size of a translation step, which is typically set at half the voxel diameter.

At the center lumen line, the magnitude of the gradient vectors becomes very small, however not exactly zero. To prevent the points of the center lumen line translating to the global maximum of the gradient distance map, we stop the translation when the magnitude of the gradient vector drops below a certain threshold.

\subsection{Vessel model}

Our vessel model is currently designed for vessel segments without changes in topology (such as bifurcations). We use Non-Uniform Rational B-Splines (NURBS) surfaces. A NURBS surface possesses many nice properties, such as 
local control of shape and the flexibility to describe both simple and complex objects. Simple surfaces can be described by less control points and the surface is smooth by construction. Complex surfaces require more control points and derivative smoothness constraints may be considered. The number of control points and the total area of the surface are not related, so the same model can work for all sizes of vessels.

\subsection{Model Matching}

The model is deformed to fit the underlying image data. To detect the lumen of the vessel we use the 3D extension of the Full Width 30\% Maximum (FW30\%M) criterion described in [9]. This method thresholds the image at $30 \%$ of the local center lumen line intensity. We adapted this criterion, by not only performing a simple threshold, but also placing restrictions on the way that the model deforms. This is done by making an energy function (3), which has to be minimized. In order to make the threshold criterion less sensitive for noise, the array of image intesities at the center lumen line in smoothed. The first step is to initialize the surface as a tube centered around the previously detected center lumen line. The second step uses the conjugate gradient algorithm to minimize equation (3). To prevent the model from intersecting itself, we restrict the movement of the control points to a plane perpendicular to the center lumen line. This restriction is sufficient in practice, because the center lumen line has no sharp bends, compared to the diameter of the vessel.

$$
\varepsilon=\varepsilon_{\text {external }}+\gamma_{s} \cdot \varepsilon_{\text {stretching }}+\gamma_{b} \cdot \varepsilon_{\text {bending }}
$$

The external energy is based on the FW30\%M criterion, and the stretching and bending energies are the internal energies that are needed to deform the NURBS-surface.

\subsection{Visualization and interaction in 3D}

We have developed a general visualization platform to visualize 3D images of any modality, and to intuitively interact with them, based on the VTK-software library [10]. It runs on a standard Windows PC, with minimal requirements of a PentiumII $500 \mathrm{MHz}, 128 \mathrm{MB}$ RAM, and an OpenGL video-card, such as a GeForce. The platform has an object oriented design that provides well known rendering techniques. We distinguish three types of data, voxel based (such as CT- and MRI-scans, as shown in Figure 2(a)), surface based (such as modelgenerated meshes, as shown in Figure 2(b)), and attribute data (such as scalars, streamlines, glyphs, vector fields, etc., as shown in Figure 2(c)). For each type of data we have developed an object that provides each of the three types of visualization: 3D rendering, projections and Multi Planar Reformatting. With these objects, a scene can be build, which can be visualized in any number of viewports, as shown in Figure 3. 


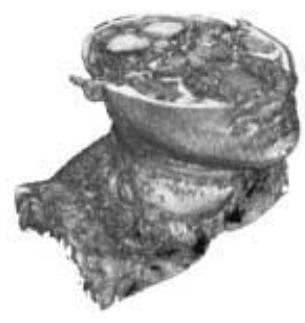

(a) Voxel data

MRA data, thresholded and then volume rendered

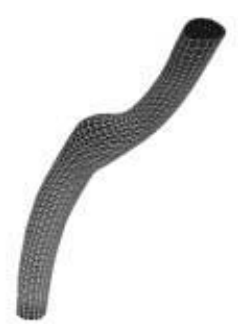

(b) Surface data

A mesh that describes the lumen

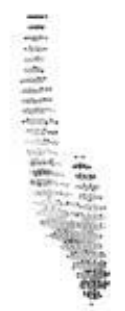

(c) Attribute data Each point represents the time that the wafefront passed

Fig. 2. Examples of the three different data types used in our visualization platform.

Initially, the data is presented using the three orthogonal MIPs and a 3D view of the same MIPs as shown in the left part of Figure 3. As stated before, the segmentation algorithm needs two points to trace the vessel. It is not possible to position a point in $3 \mathrm{D}$ with a $2 \mathrm{D}$ mouse in just one action. However, when the MIPs are calculated, the depth of the voxel with maximum intensity is stored for each ray resulting in a depth image as shown in Figure 4(a). The user can depict a point in any of the three MIPs. The depth of the depicted $2 \mathrm{D}$ coordinate is read from the depth-image, resulting in a $3 \mathrm{D}$ position. This point is then shown in all three MIPs and in a 3D view, and can be moved interactively. This method provides an intuitive and fast way to place the two seedpoints in the vessel (see Figure 4(b)).

The result of the segmentation, i.e. the centerline and the vessel wall, are visualized using surface rendering, as shown in Figure 5. The user can interactively look at these structures from all directions, and combine the objects in one or more viewports. This provides the user a better understanding of the $3 \mathrm{D}$ shape of the vessel, and of the relationship with the surrounding anatomy. In order to inspect the segmentation result more closely, Multi Planar Reformatting (MPR) can be performed to cut through the objects, resulting in $2 \mathrm{D}$ contours, which can be compared with the original image data on the plane. Again, all these actions can be done at interactive speeds and from all directions. Finally, the detected centerline can be used as a path for the camera, thus generating a fly-through inside the vessel.

\section{Validation}

The centerline detection and lumen segmentation were validated using in-vitro and in-vivo data sets. The in-vivo data was used to test the centerline detection, while the in-vitro data was used to test the lumen segmentation. The lumen 

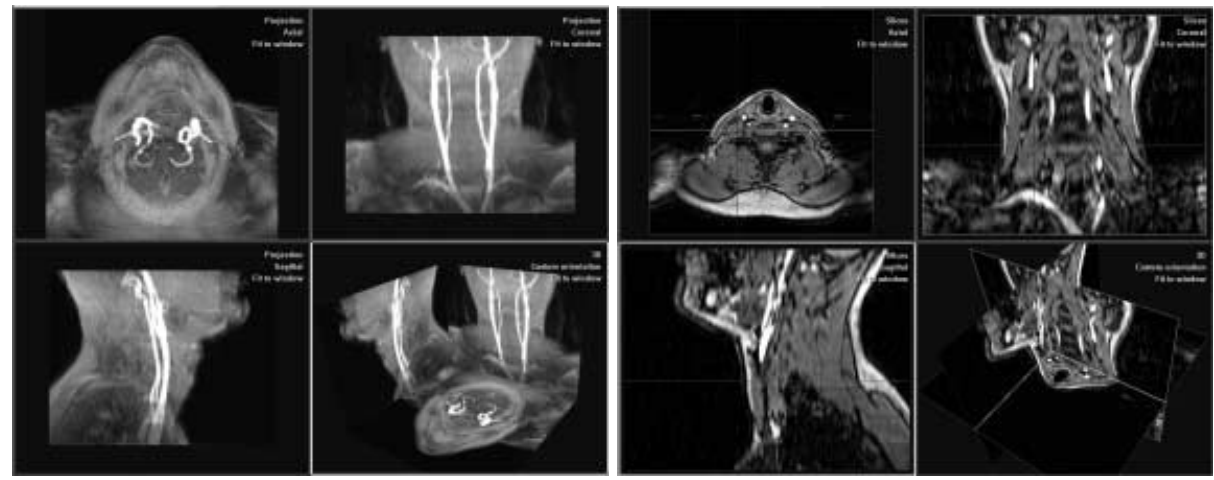

Fig. 3. The two standard screen layouts. The left figure shows three orthogonal MIPs, both in $2 D$ and $3 D$, while the right figure shows three orthogonal cross-sections, both in $2 D$ and $3 D$. When new objects are added to the scene (such as the segmented vessel), these can be viewed together in any combination of $3 D$ rendering, projections or slicing. It is also possible to have any number of viewports of any type to explore the data.

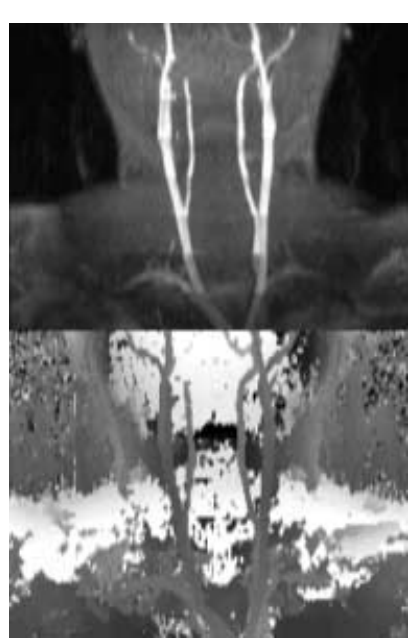

(a) MIP and its depthimage

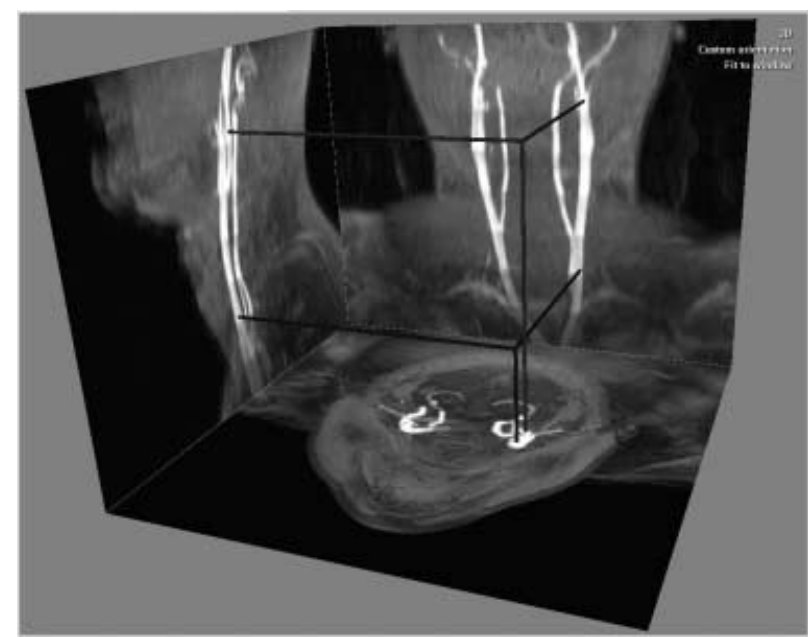

(b) The two points in $3 D$ with the three orthogonal MIPS

Fig. 4. The only needed user interaction: the user needs to depict two $3 D$ points at the beginning and the end of the vessel segment of interest. This can be done by placing a point in any of the $2 D$ orthogonal MIPs; the third coordinate is then obtained from the associated depth-image. 




Fig. 5. The segmentation result, rendered together with the MIPs of the MRA-data, the proximal and distal point of the vessel segment, and the centerline.

segmentation was also compared to conventional X-ray angiography, which is considered the current gold-standard.

\subsection{Materials}

The in-vivo data included MRA CE studies of the lower abdomen and lower extremities and MRA TOF studies of the carotid arteries. The slice thickness varied from 2.37 to $4 \mathrm{~mm}$, while the in-plane resolution varied from $0.67 \times 0.67$ to $1.76 \times 1.76 \mathrm{~mm}$.

The in-vitro data consisted of MRA Contrast Enhanced (CE) studies of several phantoms. These phantoms all had identical reference diameters and different obstruction diameters. The slice thickness was $2.37 \mathrm{~mm}$ with a slice-centerto-center-distance of $1.2 \mathrm{~mm}$, while the in-plane resolution was $1.76 \times 1.76 \mathrm{~mm}$. The morphological parameters of the phantoms are listed in table 1. Additionally X-ray angiographic (XA) images of the phantoms were acquired. Further details about the phantom and the acquisition of the data can be found in [9].

\subsection{Results}

Our center lumen line detection was validated by detecting 43 centerlines in 22 studies by 2 observers. 40 centerlines (93\%) were classified as correct (see table 2 ). The 3 failures were caused by brighter vessel running close and parallel to the vessel of interest and were identical for both observers. 
Table 1. Morphological parameters of the phantoms used in the in-vitro study.

\begin{tabular}{ccccc}
\hline $\begin{array}{c}\text { Phantom } \\
\text { number }\end{array}$ & $\begin{array}{c}\text { Reference } \\
\text { diameter }(\mathrm{mm})\end{array}$ & $\begin{array}{c}\text { Obstruction } \\
\text { diameter }(\mathrm{mm})\end{array}$ & $\begin{array}{c}\text { Percent diameter Length of stenosis } \\
\text { stenosis }(\% \mathrm{D})\end{array}$ & \begin{tabular}{c} 
(mm) \\
\hline
\end{tabular} \\
\hline \hline 1 & 6.80 & 5.58 & 18 & 7 \\
2 & 6.80 & 4.69 & 31 & 7 \\
3 & 6.80 & 3.47 & 49 & 7 \\
4 & 6.80 & 2.92 & 57 & 7 \\
5 & 6.80 & 1.97 & 71 & \\
\hline
\end{tabular}

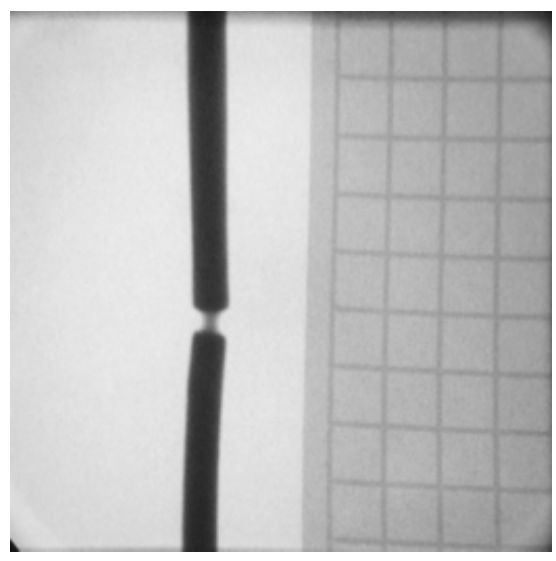

(a) X-DSA image of the phantom

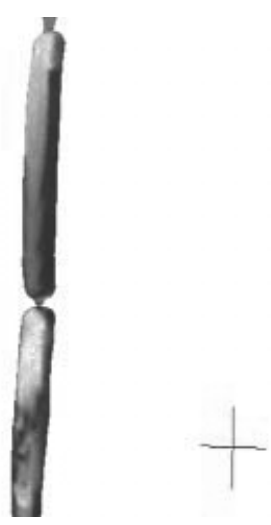

(b) MRA image of the phantom

Fig. 6. Images of the phantom.

The reference diameters of all phantoms were averaged for both MRA and XA. For the MRA data, several measurements were taken for a single phantom and averaged. See table 3 for the results.

The obstruction diameters of all phantoms were assessed and compared (see figure 8). From this figure, it becomes obvious that there is a lower bound on the diameter of the vessel that can be measured. If the diameter becomes smaller than 3 voxels, the lumen segmentation fails. If the diameter is larger then the error decreases to less than $1 \%$.

\section{Discussion}

In this paper we discussed an approach for the automated quantification of contrast enhanced MRA studies. This approach involves the detection of a center lumen line by using the Fast Marching Level Set algorithm. 
Table 2. Results of the center lumen line detection validation.

\begin{tabular}{ccccc}
\hline \multicolumn{4}{c}{ Analyst Segments } & \multicolumn{4}{c}{ Detected Classified correctly } & Success (\%) \\
\hline \hline 1 & 43 & 43 & 40 & 93.02 \\
2 & 43 & 43 & 40 & 93.02 \\
\hline
\end{tabular}

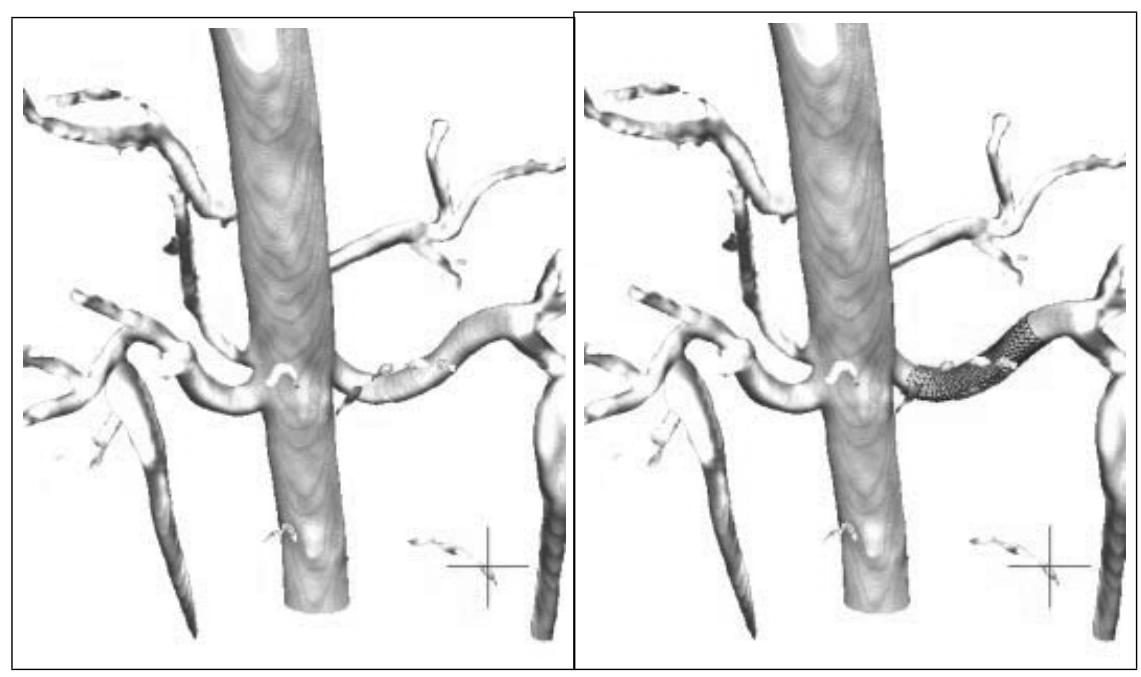

(a) Original image

(b) Segmented result and original image

Fig. 7. Images of a renal artery segmentation.

The vessel boundery detection used a model based approach. A model of a vessel was created using NURBS surfaces and then modified to minimize an energy function using the conjugate gradient methods.

Our method performs quantitative analysis bases on the original 3D images. It is known from literature [xx] that assessment of stenoses based on MIPs tends to overestimate the degree of stenosis. De Marco et al. [xx] used multi-planar reformatting (MPR) images, which allow for better visualization of the vessel lumen in a plane perpendicular to vessel axis. De Marco et al. [xx] compared stenosis grading based on MIPs and MPR images of 3D TOF MRA studies, and used intra-arterial angiography (DSA) as a standard of reference. They reported a statistically significant difference between MIPs and DSA scores with an average absolute error of $9 \%$ (SD 14\%). MPR images provided a better agreement and a negligible bias. Although this study suggests the potential benefit of MPR-based diagnosis, generation and inspection of MPRs is relatively time consuming. 


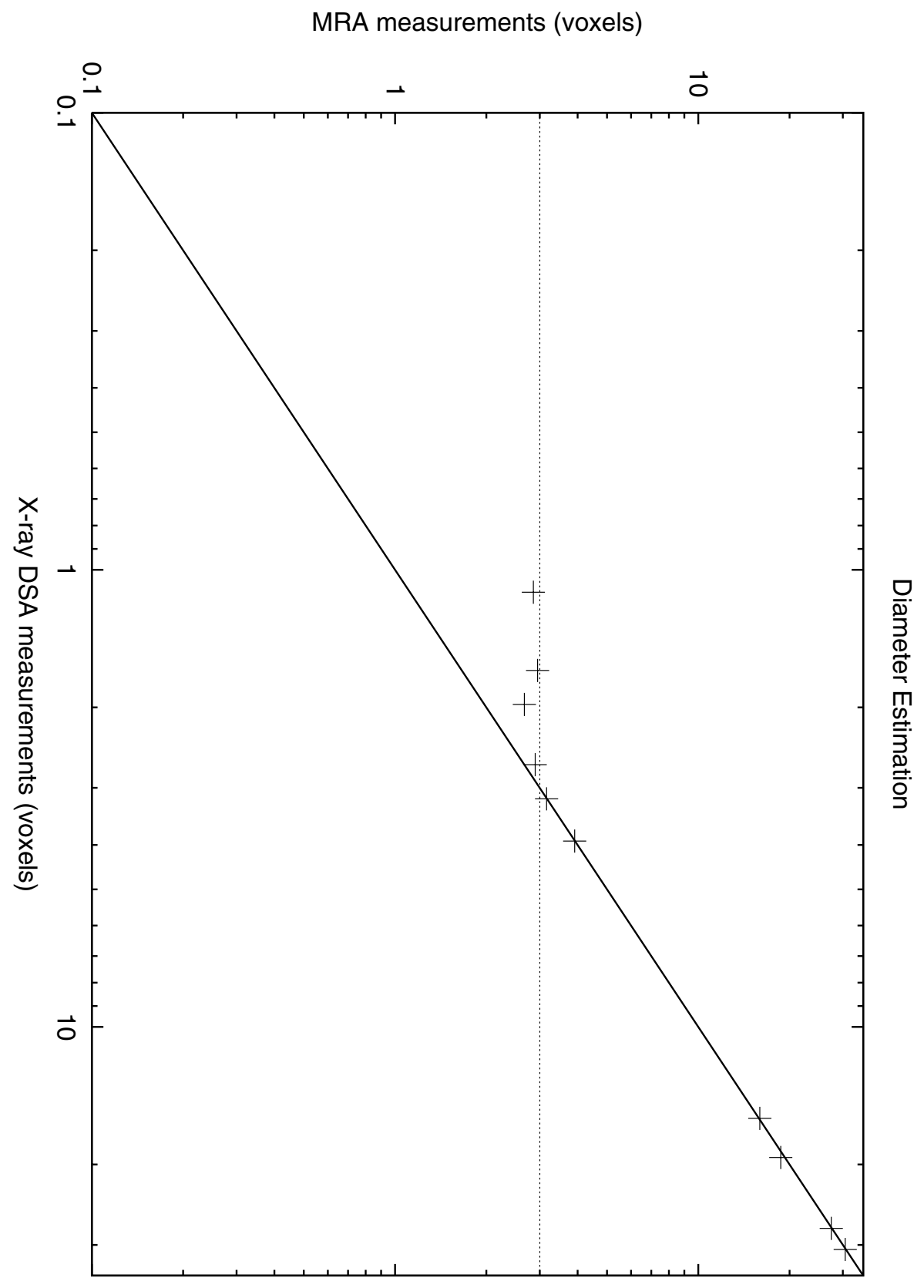

Fig. 8. Diameter estimation errors 
Table 3. Comparison X-ray DSA and MRA.

\begin{tabular}{ccc}
\hline True Diameter & X-ray DSA & MRA \\
\hline \hline $6.8 \mathrm{~mm}$ & $6.88 \pm 0.19 \mathrm{~mm} 6.87 \pm 0.25 \mathrm{~mm}$ \\
\hline
\end{tabular}

Our method shares the basic idea behind MPR based measurements. We apply an objective vessel diameter criterion in planes perpendicular to the vessel center lumen line, which is therefore similar to the radiologist's when analyzing MPR images. On the other hand, the method is objective (does not depend on window and level settings) and requires little interaction.

We used several in vivo studies to determine the accuracy and robustness of our center lumen line detection approach. In all cases a path was found, however in 3 cases the detected path was classified as incorrect by both analysts. Analysis of the cases showed a complex anatomy where several vessel were located close to each other, which resulted in a pathline which switched from one vessel to another and back again.

Using in vitro studies of a stenotic phantom, we investigated the accuracy of our vessel detection algorithm. We compared our method with X-ray DSA. The error in diameter estimation is less then $1 \%$, provided the diameter is larger then 3 voxels. This limit is inherit to the approach used, in which a center intensity is needed in order to calculate the contours. In larger vessel such as the aorta, iliac and femoral arteries this criterium is fulfilled. In smaller vessels such as the carotid arteries this criterium is not always fulfilled, especially in stenotic regions.

\section{Future Work}

Our vessel segmentation method is currently limited to vessels without bifurcations and/or side-branches. We are currently designing a more general method which incorporates bifurcations, side-branches, and specific designs for stenoses and aneurysms.

Furthermore, we will continue our collaboration with the University of Amsterdam. One of their research topics is interactive simulation [1]. We have started a joint project in which we will connect our vessel analysis package to their computer grid. The vessel geometry generated by our package will serve as input for a real time fluid dynamics simulation program. The phycisian is then enabled to try different virtual interventions and see in real time the response of the blood flow.

\section{Conclusion}

We have demonstrated that our automated vessel detection in MRA data is able to detect the vessel wall and diameter with great accuracy, provided that 
the vessel diameter is larger than three voxels. The required user-interaction is limited to placing a proximal and a distal point. Furthermore we have presented a general visualization platform that can be used to visually inspect and interact with the data and the algorithms output.

\section{References}

1. R.G. Belleman and P.M.A. Sloot. Simulated vascular reconstruction in a virtual operating theater. H.U. Lemke et al. editors, CARS Conference, Berlin, pages 938-944, 2001.

2. D. Tsuruda L.G.Shapeero C.M. Anderson, J.S. Saloner and R.E. Lee. Artifacts in maximum-intesity0projection display of mr angiograms. Amer. J. Roentgenol., 20(1):56-67, January 1990.

3. Olivier Cuisenaire. Distance Transformations: Fast Algorithms and Applications to Medical Image Processing. $\mathrm{PhD}$ thesis, Université catholique de Louvain, October 1999.

4. R. Malladi and J.A. Sethian. A real-time algorithm for medical shape recovery. In Proceedings of International Conference on Computer Vision, pages 304-310, 1998.

5. R. Malladi, J.A. Sethian, and B.C. Vemuri. Shape modeling with front propagation: A level set approach. IEEE Transactions on Pattern Analysis and Machine Intelligence, 17(2):158-175, February 1995.

6. Hartmut Schirmacher, Malte Zöckler, Detlev Stalling, and Hans-Christian Hege. Boundary surface shrinking - a continuous approach to 3D center line extraction. In B. Girod, H. Niemann, and H.-P. Seidel, editors, Proc. IMDSP '98, pages 25-28. Infix, ISBN 3-89601-011-5, 1998.

7. J. A. Sethian. A fast marching level set method for monotonically advancing fronts. Proc. of the National Academy of Sciences of the USA, 93(4):1591-1595, February 1996.

8. J. A. Sethian. Level set methods: Evolving interfaces in geometry, fluid mechanics, computer vision, and materials science. Number 3 in Cambridge monographs on applied and computational mathematics. Cambridge University Press, Cambridge, U.K., 1996. 218 pages.

9. J.J.M. Westenberg, R.J. van der Geest, M.N.J.M. Wasser, E.L. van der Linden, T. van Walsum, H.C. van Assen, A. de Roos, J. Vanderschoot, and J.H.C. Reiber. Vessel diameter measurements in gadolinium contrast enhanced three-dimensional mra of peripheral arteries. Magnetic Resonance Imaging, 18(1):13-22, January 2000 .

10. Ken Martin Will Schroeder and Bill Lorensen. The Visualization ToolKit. Prentice Hall, 2nd edition, 1998, http://www.visualizationtoolkit.org. 